\title{
AN AXIOMATIZATION OF THE ASPIRATION CORE
}

\author{
HANS KEIDING \\ Institute of Economics, University of Copenhagen \\ Studiestrcede 6, DK-1455 Copenhagen K, Denmark \\ E-mail: hans.keiding@econ.ku.dk
}

\begin{abstract}
The aspiration core of a TU game was introduced by Bennett [1] as a payoff vector which is undominated and achievable in the sense that each player belongs to a coalition which can obtain the specified payoff for its members, and which minimizes the distance to the set of aggregate feasible payoffs among all such payoff vectors. In the paper a set of axioms is proposed which characterize the aspiration core, which may be considered as an extension of the core to a much larger set of games. The axioms involve the standard notions of reduced game and converse reduced game although the notions have to be modified to allow for infeasible payoffs. Also, a class of auxiliary games which are not TU games has to be added to the domain of the abstract solution concept considered.
\end{abstract}

1. Introduction. The core of a cooperative game consists of the payoffs which cannot be improved by any coalition. Since its introduction, the core has been one of the game-theoretic solution concepts which has received most attention and has had most applications, not surprisingly in view of its intuitive appeal as well as its tractability.

The class of games for which this solution concept is meaningful, in the sense that the core is nonempty, was characterized in the seminal papers by Bondareva [2] and Shapley [11] as the family of balanced games. On the other hand, in many situations there is a need for a solution concept which agrees with the core on the family of balanced games but takes nonempty values also on games which are not balanced. Such solution concepts are core extensions; in the literature they come in two different versions, namely either (i) as solutions in the strict sense, selecting payoffs that are feasible (can be effectuated by the grand coalition), or (ii) aspiration payoffs which are not necessarily feasible but still represent realistic expectations of each player as to what can be achieved in the game.

In the present paper, we consider the second version of core extensions; we present an axiomatic characterization of the aspiration core, a core extension introduced by Bennett

2000 Mathematics Subject Classification: 91A12.

Key words and phrases: aspiration set, aspiration core, reduced game properties.

The paper is in final form and no version of it will be published elsewhere. 
[1] which assigns to each game a set of aspiration payoffs which coincides with the core when the latter is nonempty and captures most of the features of the core (with the obvious exception of overall feasibility) when the core is empty. The axioms are largely the standard ones (including a version of the consistency axiom used in most axiomatizations), and the approach follows at least initially that of Moldovanu and Winter [7] giving an axiomatic characterization of the larger aspiration set, but in order to capture the specific feature of the aspiration core consisting in minimization of distance to the feasible set, we need to bring into our discussion a class of games which strictly speaking are not TU (transferable utility) games but which arise naturally in our context, and to which the standard axioms are readily extended.

The paper is structured as follows: Section 2 contains the necessary definitions, and in Section 3 we present the basic axioms, narrowing down the solution concept to a subset of the aspiration set. Then in Section 4 we introduce the family of almost TU games and consider the core of an almost TU game. This is applied in Section 5 to give the full axiomatic characterization of the aspiration core; Section 6 contains some final remarks on the approach.

2. Definitions. In this section we introduce the basic notions which will be used throughout this paper. A cooperative transferable utility game (shorthand a TU game or just a game) is a pair $(N, v)$, where $N$ is a non-empty, finite set of players, and $v$ a function assigning to each non-empty subset (coalition) $S$ of $N$ a number $v(S)$ interpreted as the worth of the coalition $S$, an amount available for division between the members of the coalition. With the notation $\mathcal{P}_{0}(N)$ for the set of nonempty subsets of $N, v: \mathcal{P}_{0}(N) \rightarrow \mathbb{R}$ is called the characteristic function of the game $(N, v)$. Let $\mathcal{G}(N)$ denote the set of all TU games with player set $N$, and let $\mathcal{G}$ be the set of all TU games.

If $(N, v)$ is a game, $a>0$ a positive real number, and $b \in \mathbb{R}^{N}$, then the game $a v+b$ is defined by $(a v+b)(S)=a v(S)+\sum_{i \in S} b_{i}$ for each $S \in \mathcal{P}_{0}(N)$. We use the notation $\lambda A$ for the set $\{\lambda a \mid a \in A\}$, where $\lambda \in \mathbb{R}$ and $A \subset \mathbb{R}^{d}$ for some $d \in \mathbb{N}$. Finally, $A+B$ denotes Minkowski addition of sets $\left(\right.$ in $\left.\mathbb{R}^{d}\right)$.

We shall be considering TU games throughout this paper, but occasionally we shall need also the concept of an NTU game: A cooperative non-transferable utility game is a pair $(N, V)$, where as before, $N$ is a nonempty finite set of players, and for each $S \in \mathcal{P}_{0}(N), V(S)$ is a subset of $\mathbb{R}^{S}$, such that for each $S, V(S)$ is non-empty, closed, comprehensive (i.e., if $x \in V(S), y \in \mathbb{R}^{S}$, and $y^{i} \leq x^{i}$ for all $i \in S$, then $y \in V(S)$ ), and satisfies $\left.V(S) \neq \mathbb{R}^{S}\right)$. Clearly, a TU game $(N, v)$ may be viewed as an NTU game $(N, V)$ where for each $S, V(S)=\left\{x \in \mathbb{R}^{S} \mid \sum_{i \in S} x_{i} \leq v(S)\right\}$.

We now return to the context of TU games. A payoff vector of the game $(N, v)$ is an element $x$ of $\mathbb{R}^{N}$; it is feasible if $\sum_{i \in N} x_{i} \leq v(N)$, and individually rational if $x_{i} \geq \max V(\{i\})$ for each $i \in N$; more generally, a payoff vector is dominated via the coalition $S \in \mathcal{P}_{0}(N)$ if $\sum_{i \in S} x_{i}<v(S)$. The core of $(N, v)$, written $\mathrm{C}(N, v)$, is the set of payoffs $x$ such that

(i) $\sum_{i \in N} x_{i}=v(N)$,

(ii) $x$ is undominated. 
As is well-known, the subclass $\mathcal{G}_{b}$ of $\mathcal{G}$ consisting of games $(N, v)$ for which $\mathrm{C}(N, v) \neq \emptyset$, consists of the balanced games: A subfamily $\mathcal{B}$ of $\mathcal{P}_{0}$ is said to be balanced if there are nonnegative numbers $\lambda_{B}, B \in \mathcal{B}$ (the balancing weights of $\mathcal{B}$ ) such that $\sum_{B \in \mathcal{B}} \lambda_{B} 1_{B}=1_{N}$ (here, $1_{S}$ denotes the vector in $\mathbb{R}$ with $i$ th coordinate 1 if $i \in S$ and 0 otherwise), and a game $(N, v)$ is balanced if

$$
\sum_{B \in \mathcal{B}} \lambda_{B} v(B) \leq v(N)
$$

for each balanced family $\mathcal{B}$ with balancing weights $\left(\lambda_{B}\right)_{B \in \mathcal{B}}$.

Let $\mathcal{G}^{\prime} \subseteq \mathcal{G}$. A solution concept on $\mathcal{G}^{\prime}$ is a map $\Phi$ which to every game $(N, v) \in \mathcal{G}^{\prime}$ assigns a nonempty subset of $\mathbb{R}^{N}$. Thus, the core is a solution concept on $\mathcal{G}_{b}$ but not on $\mathcal{G}$. A solution concept defined on any $\mathcal{G}^{\prime}$ containing $\mathcal{G}_{b}$ such that

$$
\Phi(N, v)=\mathrm{C}(N, v) \text { for }(N, v) \in \mathcal{G}_{b}
$$

is called a core extension.

An example of a core extension is the Gomez extension (cf. Gomez [3]) $\Phi_{G}$ defined on all $(N, v) \in \mathcal{G}$ such that $v(S) \geq 0$ for all $S$, with

$$
\Phi_{G}(N, v)=k(N, v)^{-1} C\left(N, v_{k(N, v)}\right),
$$

where $\left(N, v_{k}\right)$ for $k \geq 0$ is defined as the game with $v_{k}(S)=v(S)$ for $S \neq N, v_{k}(N)=$ $k v(N)$, and $k(N, v)$ is the smallest $k \geq 0$ such that $\mathrm{C}(N, v) \neq \emptyset$. As it can be seen from the definition, the Gomez extension first chooses a core element in a suitably chosen balanced game resembling the given one, and secondly maps the core elements of this game to feasible payoffs of the original game.

In the present paper we shall be interested in extensions which perform only the first of the two steps mentioned above, so that it selects core elements from a suitably chosen balanced game. The demand that this game should be closely related to the original one may be further specified to leaving unchanged $v(S)$ for every $S \subsetneq N$, and we are then led to subsets of the aspiration set as introduced by Bennett [1] and reconsidered in the context of NTU games as semi-stable demand vectors by Moldovanu and Winter [7].

For $(N, v) \in \mathcal{G}$ a game, define the aspiration set $\operatorname{Asp}(N, v)$ as the set of vectors $x \in \mathbb{R}^{N}$ which satisfy

(i) for each $i \in N$ there is $S \in \mathcal{P}_{0}(N)$ with $i \in S$ such that $\sum_{j \in S} x_{j} \leq v(S)$,

(ii) for each $S \in \mathcal{P}_{0}(N), \sum_{i \in S} x_{i} \geq v(S)$.

While the last condition states that $x$ should be undominated, a notion which has already been introduced, the first condition, that each $i$ belongs to a coalition $S$ which can attain $\left(x_{i}\right)_{i \in S}$, introduces a weaker notion of feasibility than the standard one (that the payoff vector should be attainable by $N$ ); here we demand only that the payoff vector is realistic in the sense that each player might attain her payoff in some coalition.

Before we proceed, we notice the following simple but nevertheless useful fact.

Lemma 1. Let $x, x^{\prime} \in \operatorname{Asp}(N, v), x \neq x^{\prime}$. Then there is at least one $i \in N$ such that $x_{i}>x_{i}^{\prime}$.

Proof. Assume to the contrary that $x_{i}^{\prime} \geq x_{i}$ for all $i$. Let $i$ be such that $x_{i}^{\prime}>x_{i}$ and choose a coalition $T$ containing $i$ such that $\sum_{i \in T} x_{i}^{\prime} \leq v(T)$. Then $\sum_{i \in T} x_{i}<\sum_{i \in T} x_{i}^{\prime} \leq v(T)$, so that $x$ is dominated via $T$, contradicting that $x$ belongs to $\operatorname{Asp}(N, v)$. 
We may now define a central concept of the present paper, namely the aspiration core $\mathrm{AC}(N, v)$, defined as the set of elements of $\operatorname{Asp}(N, v)$ for which $\sum_{i \in N} x_{i}$ is minimal. The aspiration core extends the core in the sense that for all $(N, v) \in \mathcal{G}_{b}, \mathrm{AC}(N, v)=\mathrm{C}(N, v)$; indeed, $\mathrm{C}(N, v)$ belongs to the aspiration set and trivially minimizes $\sum_{i \in N} x_{i}$ on this set.

The idea of considering payoffs which are not feasible in the strict sense is not new. The aspiration set was introduced in Bennett [1] with the interpretation as player anticipations of the payoffs to be obtained, and the aspiration core was introduced under the notion of a balanced aspiration. The following result is due to Bennett:

Lemma 2. Let $(N, v) \in \mathcal{G}$. Then $A C(N, v) \neq \emptyset$.

Proof. Let $(N, V)$ be the NTU game defined by

$$
\begin{aligned}
& V(S)=\left\{\left(x_{i}\right)_{i \in S} \mid \sum_{i \in S} x_{i} \leq v(S)\right\} \text { for } S \subsetneq N \\
& V(N)=\left\{x \in \mathbb{R}^{N} \mid \forall i \in N \exists S \in \mathcal{P}_{0}(N), \sum_{i \in S} x_{i} \leq v(S)\right\} .
\end{aligned}
$$

Let $\mathcal{B}$ be a balanced family of coalitions and suppose that $x \in \cup_{S \in \mathcal{B}} V(S)$. Then for each $i$, there is $S \in \mathcal{B}$ such that $i \in S$; consequently, if $\left(x_{i}\right)_{i \in S} \in V(S)$ for all $S$, then $x$ satisfies condition (i) in the definition of $\operatorname{Asp}(N, v)$, or equivalently, $x \in V(N)$. From standard results (e.g. Scarf, [10]) on the nonemptieness of the core of NTU games it follows that $\mathrm{C}(N, V) \neq \emptyset$. By our construction of $(N, V)$, this means that $\operatorname{Asp}(N, v) \neq \emptyset$. Since $\operatorname{Asp}(N, v)$ is closed and bounded from below, the result follows.

3. Axioms for the aspiration core: Standard axioms. In this section, we introduce a set of axioms which characterize the aspiration core. As a beginning, we consider axioms which are rather uncontroversial and which have proved useful in axiomatizations of the (ordinary) core. Since this does not give us a full system of axioms, we add some further axioms in the following sections.

Formally, we are concerned with maps $\Phi: \mathcal{G}^{\prime} \rightarrow \mathcal{P}_{0}\left(\mathbb{R}^{N}\right)$, where $\mathcal{G}^{\prime}$ is a set of games containing $\mathcal{G}_{b}$. A natural place to start is the following:

Axiom 1 (Triviality). Let $(N, v) \in \mathcal{G}^{\prime}$ be a trivial game, i.e. $v(S)=0$ for all $S \subset N$. Then $\Phi(N, v)=\{\mathbf{0}\}$.

Thus, if we are given a conflict situation where no individual or coalition can attain anything for its members, then the solution concept assigns to this game the zero vector $\mathbf{0}$ as the only possible payoff.

The next axiom is similarly uncontroversial and has to do with changes of scale and origin for measuring the payoffs of the players.

Axiom 2 (Invariance under affine transformations). Let $(N, v) \in \mathcal{G}^{\prime}$, and let $a>0 b \in \mathbb{R}^{N}$ be arbitrary. Then $(N, a v+b) \in \mathcal{G}^{\prime}$, and $\Phi(N, a v+b)=a \Phi(N, v)+b$.

Here, the change of scale is the same for all players, whereas the individual displacements of the origin may differ. The solution should not be essentially effected by such changes. 
Now we turn to axioms that are less intuitively appealing but on the other hand more powerful in restricting the set of possible solutions. We shall need some version of consistency, which in its turn requires the construction of a reduced game. We follow here the approach of Moldovanu and Winter [7], which takes into account that payoff vectors may not be feasible.

Let $(N, v) \in \mathcal{G}^{\prime}$ be a game, let $S \in \mathcal{P}_{0}(N)$ be a coalition and let $x \in \mathbb{R}^{N}$ be a payoff vector (not necessarily feasible) The reduced game of $(N, v)$ w.r.t. $S$ and $x$ is the game $\left(S, v^{S, x}\right)$ defined by

$$
v^{S, x}(T)=\max _{Q \subseteq N \backslash S}\left[v(T \cup Q)-\sum_{i \in Q} x_{i}\right], \text { all } T \subseteq S .
$$

With this notion of a reduced game we may introduce the following axiom.

Axiom 3 (MW-consistency). Let $(N, v) \in \mathcal{G}^{\prime}, S \in \mathcal{P}_{0}(N)$, and $x \in \Phi(N, v)$. Then $\left(S, v^{S, x}\right) \in \mathcal{G}^{\prime}$, and $x_{S} \in \Phi\left(S, v^{S, x}\right)$.

The consistency property has a long record of use in axiomatizations of game theoretic solution concepts. It was used (again in a slightly different form) for the characterization of the core by Peleg [8].

The usefulness of the axioms formulated above can be seen from the following result (first established by Gomez [4]).

Lemma 3. The aspiration core satisfies Axioms 1-3. Conversely, if a solution concept $\Phi$ on $\mathcal{G}$ satisfies Axioms 1-3, then $\Phi(N, v) \subseteq \operatorname{Asp}(N, v)$ for every $(N, v) \in \mathcal{G}$.

Proof: Let $x \in \Phi(N, v)$. Assume first that $(N, v)$ is a one-player game, that is $N=\{i\}$. Then it may be considered as a transformation of a trivial game, and consequently by Axiom 1 and $2, \Phi(\{i\}, v)=v(\{i\})$. In particular, for arbitrary $(N, v) \in \mathcal{G}$ we have that $\Phi\left(\{i\}, v^{\{i\}, x}\right)=v^{\{i\}, x}(\{i\})$. By Axiom $3, x_{i} \in \Phi\left(\{i\}, v^{\{i\}, x}\right)$, so $x_{i}=v^{\{i\}, x}(\{i\})$.

Let $S \in \mathcal{P}_{0}(N)$. Then, using the definition of the reduced game we obtain that

$$
x_{i}=v^{\{i\}, x}(\{i\}) \geq v(\{i\} \cup[S \backslash\{i\}])-\sum_{j \in S \backslash\{i\}} x_{i},
$$

and rearranging, we have that $\sum_{i \in S} x_{i} \geq v(S)$, which is (ii) in the definition of $\operatorname{Asp}(N, v)$. To show that also (i) is fulfilled, we note that for each $i \in N$ there is $T \in \mathcal{P}_{0}(N)$ with $i \in T$ such that $\sum_{i \in T} x_{i}=v(T)$. Since $x_{i}=v^{\{i\}, x}(\{i\})$, there must be $Q \subseteq N \backslash\{i\}$ such that $x_{i}=v(\{i\} \cup Q)-\sum_{j \in Q} x_{j}$, and setting $T=\{i\} \cup Q$, we have the desired conclusion.

4. Almost TU games. In this section, we introduce a class of games which are not TU but on the other hand differ from TU games only with regard to the set of feasible payoffs (of the grand coalition). Formally, an NTU game $(N, W)$ is almost TU if there is a function $w: \mathcal{P}_{0}(N) \backslash\{N\} \rightarrow \mathbb{R}$ such that $W(S)=\left\{x_{S} \in \mathbb{R}^{S} \mid \sum_{i \in S} x_{i} \leq w(S)\right\}$ for all $S \subsetneq N$, whereas $W(N)$ does not necessarily have this form. We write the almost TU game as $(N, W, w)$ when we need to emphasize the function $w$. The class of almost TU games, denoted $\widetilde{\mathcal{G}}$, will be used in our axiomatization of the aspiration core, and the purpose of this section is to establish some results about the core and its axiomatization for this class. 
For this, we introduce the reduced game of an almost TU game: Let $(N, W, w)$ be almost TU, let $S \subsetneq N$ and let $x \in W(N)$. Then the reduced game of $(N, W, w)$ w.r.t. $S$ and $x$ is defined by

$$
\begin{aligned}
& W^{S, x}(S)=\left\{\left(x_{S}^{\prime} \mid\left(x_{S}^{\prime}, x_{N \backslash S}\right) \in W(N)\right\},\right. \\
& W^{S, x}(T)=\left\{x_{T}^{\prime} \mid \exists Q \subset N \backslash S,\left(x_{T}^{\prime}, y_{Q}\right) \in W(T \cup Q)\right\}, \text { for } T \subsetneq S,
\end{aligned}
$$

that is, by the standard definition of a reduced game of an NTU game (cf. Peleg and Südhölter [9]). It is easily seen that the reduced game of an almost TU game is again almost TU (so that the property of being almost TU is hereditary).

An almost TU game is said to be nonlevel if for each $x, y \in \partial W(N)$ (the boundary of $W(N))$, the inequality $x \geq y$ implies $x=y$. If $(N, W)$ is nonlevel, then so is any reduced game $\left(N, W^{S, x}\right)$. The core of an almost TU game, $C(N, W)$, consists of all $x \in W(N)$ such that no coalition $S \in \mathcal{P}_{0}(N)$ dominates $x$, which for nonlevel almost TU games $(N, W, w)$ means that $x \in \partial W(N)$ and $\sum_{i \in S} x_{i} \geq w(S)$ for each $S \subsetneq N$. If $x \in C(N, W)$, and $S \subseteq N$ is arbitrary, then clearly $x_{S} \in C\left(S, W^{S, x}\right)$, so the family of almost TU games with nonempty core is stable under reduction.

The notion of a solution concept extends to the class of almost TU games; thus a solution concept on a family $\widetilde{\mathcal{G}}$ of almost TU games is a map $\Phi$ which to each $(N, W, w)$ in $\widetilde{\mathcal{G}}$ assigns a nonempty subset of $\mathbb{R}$, and the Axioms 1,2 are meaningful also for such extended solution concepts. We restate the reduced game property introduced in the previous section, since we use the ordinary definition of reduction for almost TU games rather than the MW-reduction.

Axiom 4 (Consistency). Let $(N, W) \in \widetilde{\mathcal{G}}_{b}$, let $S \subset N$, and let $x \in \Phi(N, W)$. Then $x_{S} \in \Phi\left(S, W^{S, x}\right)$.

In our application of almost TU games, we shall need that the solution concept $\Phi$ coincides with the core on $\widetilde{\mathcal{G}}_{b}$. To this purpose we might use an axiom stating some property of converse consistency (such as the reconfirmation property, cf. Hwang and Südhölter [6]). To simplify proofs, we use the following less elegant but rather powerful property:

Axiom 5 (Monotonicity). Let $(N, W),\left(N, W^{\prime}\right) \in \widetilde{\mathcal{G}}_{b}$, and assume that $W(N)=W^{\prime}(N)$, $W(S) \subseteq W^{\prime}(S)$ for all $S \subset N$. Then $\Phi\left(N, W^{\prime}\right) \subset \Phi(N, W)$.

We now have the following:

LEMma 4. Assume that $\Phi$ is a solution concept defined on the set $\widetilde{\mathcal{G}}_{b}$ of all almost $T U$ games with nonempty core. If $\Phi$ satisfies Axiom 1, 2, and 4, then $\Phi(N, W) \subseteq \mathrm{C}(N, W)$ for each $(N, W) \in \tilde{\mathcal{G}}_{b}$. If, in addition, $\Phi$ satisfies Axiom 5, then $\Phi(N, W)=\mathrm{C}(N, W)$ for all $(N, W) \in \widetilde{\mathcal{G}}_{b}$.

Proof. Let $x \in \Phi(N, W)$. Arguing as in the proof of Lemma 3, by Axioms 1 and 2 we have that for each $i \in N, \Phi\left(N, W^{N \backslash\{i\}, x}\right)=\max W^{\{i\}, x}(\{i\})$, and by Axiom 3, we therefore get that $x_{i}=\max W^{\{i\}, x}(\{i\})$; in particular, $x$ is Pareto optimal in $W(N)$ (that is, if $y \in W(N)$ and $y_{i} \geq x_{i}$, all $i$, then $y=x$ ). If $S \subseteq N$, then $\sum_{i \in S} x_{i} \geq w(S)$ (again following the reasoning of Lemma 3 ), so that no proper subcoalition of $N$ dominates $x$. It follows that $x$ belongs to the core of $(N, W)$. 
Next, let $(N, W, w) \in \widetilde{\mathcal{G}}_{b}, x \in \mathrm{C}(N, W)$. We must show that $x \in \Phi(N, W)$. Define a new almost TU game $\left(N, W^{\prime}\right)$ by $W^{\prime}(\{i\})=\left\{x_{i}^{\prime} \mid x_{i}^{\prime} \leq x_{i}\right\}$ for each $i \in N, W^{\prime}(S)=W(S)$ for all $S \in \mathcal{P}_{0}(N)$ with $|S|>1$. Then $\left(N, W^{\prime}\right) \in \widetilde{\mathcal{G}}_{b}$, and $(N, W)$ is nonlevel, so by Lemmas 1 and 2 we have that $x$ is the unique core element of $\left(N, W^{\prime}\right)$. By the first part of the proof, $x \in \Phi\left(N, W^{\prime}\right)$, and using Axiom 5 we now get that $x \in \Phi(N, W)$.

5. Axioms for the aspiration core II: The debt-extended game. In Section 3, we have considered a family of axioms which are rather uncontroversial but on the other hand are not sufficient to characterize the aspiration core; we lack a property which reflects the choice of a minimal element of the aspiration set. This may be remedied by being explicit on the fact that the solution concept may choose non-feasible payoffs, whereby the set of all players (the "grand coalition") gets into a position as net debtor to its environment.

Let $(N, v)$ be a TU-game, and define the d-extended game to be the NTU game $\left(N \cup\{*\}, W_{d, v}\right)$, where the characteristic function $W_{d, v}: \mathcal{P}_{0}\left(N \cup\{*\} \rightarrow \mathcal{P}\left(\mathbb{R}^{N+1}\right)\right.$ is defined by

$$
W_{d, v}(N \cup\{*\})=\left\{\left(x, x_{*}\right) \mid x \in \operatorname{Asp}(N, v), \sum_{i \in N} x_{i}+x_{*} \leq v(N)\right\}
$$

for the grand coalition $N \cup\{*\}$, and $W_{d, v}(T)=\left\{x_{T} \mid \sum_{i \in T} x_{i} \leq v(T)\right\}$ if $T \subseteq N$,

$$
\begin{aligned}
W_{d, v}(T)=\left\{\left(x_{T}, x_{*}\right) \mid\right. & \exists\left(x^{\prime}, x_{*}^{\prime}\right) \in W_{d, v}(N), \\
& \left.\sum_{i \in T} x_{i}^{\prime} \leq v(T \backslash\{*\}), \sum_{i \in T} x_{i}+x_{*} \leq \sum_{i \in T} x_{i}^{\prime}+x_{*}^{\prime}\right\}
\end{aligned}
$$

if $T \subsetneq N$.

Thus, the d-extended game involves a new player, denoted $*$, holding the debt contracted by the coalition $N$ in order to achieve a payoff in $\operatorname{Asp}(N, v)$, which therefore is achievable by the players of $N$ in the game $\left(N \cup\{*\}, W_{d, v}\right)$. The original coalitions $T \subset N$ can achieve the same payoffs in $\left(N \cup\{*\}, W_{d, v}\right)$ as they could in $(N, v)$, but cannot contract debt, whereas coalitions containing $*$ can contract debt which however must not exceed what can occur at payoffs belonging to $\operatorname{Asp}(N, v)$ and sustainable by the coalition. It should be noted that the game $\left(N \cup\{*\}, W_{d, v}\right)$ is almost $\mathrm{TU}$ in the sense that for $S \subsetneq N$, if $x_{S} \in W_{d, v}(S)$, then so is any $x_{S}^{\prime}$ such that $\sum_{i \in S} x_{i}^{\prime}=\sum_{i \in S} x_{i}$ (whether or not the new player $*$ belongs to the coalition $S$ ). It is, however, important for the construction that the transferability of utility does not extend to the new, artificial player in the set of feasible (for $N \cup\{*\}$ ) payoffs.

Intuitively, the d-extension amounts to including an artificial outside "creditor" whose payoff at some vector $x$ in the original game is minus the amount by which $\sum_{i \in N} x_{i}$ exceeds $v(N)$ (the "debt" at $x$ ). This artificial player can of course block feasible outcomes $x$ in $\left(N, W_{d, v}\right)$ for which the "debt" is larger than it is at $\mathrm{AC}(N, v)$, thus providing a link between this solution concept and the core, albeit in another context. The d-extended game is not a TU game, but it is an almost TU game, and moreover, by Lemma 1, it is nonlevel. The connection between the original TU game $(N, v)$ and the d-extended game $\left(N, W_{d, v}\right)$ is considered in the following lemma. 
Lemma 5. Let $(N, v)$ be a TU game, and assume that $x \in \operatorname{Asp}(N, v)$ belongs to the aspiration core of $(N, v)$. If $S \subset N$ is arbitrary, then $\left(x^{S}, x_{*}\right)$ belongs to the core of $\left(S \cup\{*\}, W_{d, v}^{S \cup\{*\},\left(x, x_{*}\right)}\right)$, the d-reduced game of $(N, v)$ w.r.t. $S$ and $x$.

Proof. Assume to the contrary that $\left(x^{S}, x_{*}\right)$ does not belong to the core of $\left(N \cup\{*\}, W_{d, v}^{S \cup\{*\},\left(x, x_{*}\right)}\right)$. We show that this leads to a contradiction.

First of all, it follows trivially from our construction that

$$
\left(x^{S}, x_{*}\right) \in W_{d, v}^{S \cup\{*\},\left(x, x_{*}\right)}(S \cup\{*\}),
$$

so that $\left(x^{S}, x_{*}\right)$ is feasible. Suppose that there is a coalition $T$ which dominates $\left(x^{S}, x_{*}\right)$ in the game $\left(N \cup\{*\}, W_{d, v}^{S \cup\{*\},\left(x, x_{*}\right)}\right)$. Then either (i) $T \subset N$, and from

$$
\left(x_{i}^{S}\right)_{i \in T} \in \operatorname{int} W_{d, v}^{S \cup\{*\},\left(x^{S}, x_{*}\right)}(T)
$$

we get that $\sum_{i \in T} x_{i}^{S}<v(T)$ showing that $T$ dominates $x$, contradicting that $x$ belongs to the aspiration core, or (ii) $* \in T$; in this case, there must be $Q \subset N \backslash S$ such that

$$
\left(\left(x_{i}^{S}\right)_{i \in T \cup Q}, x_{*}\right) \in \operatorname{int} W_{d, v}^{S \cup\{*\},\left(x^{S}, x_{*}\right)}(T \cup Q)
$$

we get that there is $\left(x^{\prime},\left(x^{\prime}\right)_{*}\right) \in \operatorname{asp}(N, v)$ such that

$$
\sum_{i \in(T \cup Q) \backslash\{*\}} x_{i}<\sum_{i \in(T \cup Q) \backslash\{*\}} x_{i}^{\prime}<v(T \cup Q \backslash\{*\})
$$

and $\left(x^{\prime}\right)_{*}>x_{*}$, a contradiction.

The d-reduced game is admittedly a somewhat tedious construction, but it turns out useful in the characterization of the aspiration core.

Lemma 6. Let $(N, v)$ be a TU game, and let $x$ be a payoff which is in the aspiration set $\operatorname{Asp}(N, v)$ and is undominated but does not belong to the aspiration core. Then there is $S \subset N$ such that $\left(\left(x_{i}\right)_{i \in T}, x_{*}\right)$ does not belong to the core of $\left(S \cup\{*\}, W_{d, v}^{S \cup\{*\},\left(x, x_{*}\right)}\right)$.

Proof. By our assumptions, there exists $x^{\prime} \in \operatorname{Asp}(N, v)$ such that $x^{\prime}$ is undominated and $\left(x^{\prime}\right)_{*}>x_{*}$. Let $T \subset N$ be such that $\sum_{i \in T} x_{i}^{\prime}=v(T)$. Then $T \neq N$; let $S=N \backslash T$ and consider the d-reduced game $\left(S \cup\{*\}, W_{d, v}^{S \cup\{*\},\left(x, x_{*}\right)}\right)$. By definition of the d-reduction, we have that

$$
\left(x^{\prime}\right)_{*} \in W_{d, v}^{S \cup\{*\},\left(x, x_{*}\right)}(\{*\}),
$$

(since $\left(\left(x_{i}^{\prime}\right)_{i \in T},\left(x^{\prime}\right)_{*}\right)$ belongs to $\left.W_{d, v}(T \cup\{*\})\right)$, consequently $\left(\left(x_{i}\right)_{i \in S}, x_{*}\right)$ is dominated via $\{*\}$ in $\left(S \cup\{*\}, W_{d, v}^{S \cup\{*\},\left(x, x_{*}\right)}\right)$ and therefore does not belong to its aspiration core.

We may now return to the problem of axiomatizing the aspiration core of a TU game. The approach to be followed will be to transfer the problem to $\widetilde{\mathcal{G}}_{b}$ and use the results obtained in the previous sections. For this, we need a final axiom connecting the solutions chosen on $\mathcal{G}$ and on $\widetilde{\mathcal{G}}_{b}$; it may be thought of as an extension of the consistency requirements already specified in Axioms 4 and 5.

Axiom 6. Let $\Phi$ be a solution concept on $\mathcal{G}^{\prime} \cup \widetilde{\mathcal{G}}_{b}^{\prime}$ with $\mathcal{G}_{b} \subset \mathcal{G}^{\prime}$. If $(N, v) \in \mathcal{G}$ and $x \in \Phi(N, v)$, then $\left(x, x_{*}\right) \in \Phi\left(N, W_{d, v}\right)$.

Now we may state and prove the main results: 
THEOREM 1. Let $\Phi$ be a solution concept on $\mathcal{G}^{\prime} \cup \widetilde{\mathcal{G}}_{b}^{\prime}$ with $\mathcal{G}_{b} \subset \mathcal{G}^{\prime}$. If $\Phi$ satisfies Axioms 1-6, then $\Phi(N, v)=\mathrm{AC}(N, v)$ for every $(N, v) \in \mathcal{G}^{\prime}$.

Proof. Assume that $\Phi$ satisfies Axioms 1-3 and 5. Let $(N, v) \in \mathcal{G}$ be arbitrary, and let $x \in \Phi(N, v)$. We claim that $x \in \operatorname{Asp}(N, v)$.

For each $i \in N$, consider the game $\left(\{i\}, v^{\{i\}, x}\right)$ is a game with only one player. Using Axiom 1 and 2, we get that $\Phi\left(\{i\}, v^{\{i\}, x}\right)=v^{\{i\}, x}(\{i\})$. From Axiom 3 we have that $x_{i} \in \Phi\left(\{i\}, v^{\{i\}, x}\right)$, so $x_{i}=v^{\{i\}, x}(\{i\})$.

Next, let $S \subset N$ be arbitrary. For each $i \in S$ we have that

$$
x_{i}=v^{\{i\}, x}(\{i\}) \geq v(\{i\} \cup[S \backslash\{i\}])-\sum_{i \in S \backslash\{i\}} x_{i},
$$

and rearranging we have that $\sum_{i \in S} x_{i} \geq v(S)$, which is condition (ii) in the definition of $\operatorname{Asp}(N, v)$. To show that also condition (i) is fulfilled, choose $i \in N$ arbitrarily. Since $x_{i}=v^{\{i\}, x}$, by the definition of the reduced game there must be some coalition $Q \in$ $\mathcal{P}_{0}(N \backslash\{i\})$ such that $x_{i}=v(\{i\} \cup Q)-\sum_{j \in Q} x_{j}$, and clearly $T=Q \cup\{i\}$ contains $i$ and satisfies $\sum_{i \in T} x_{i} \leq v(T)$. This proves our claim.

To prove that $x \in \mathrm{AC}(N, v)$, we assume on the contrary that $x$ does not belong to the aspiration core of $(N, v)$. Then by Lemma 6 , we have that there is $S \subsetneq N$ such that $\left(x_{S}, x_{*}\right)$ does not belong to the core of the d-reduced game $\left(S \cup\{*\}, v^{S \cup\{*\},\left(x, x_{*}\right)}\right)$. On the other hand, the d-reduced game belongs to $\widetilde{\mathcal{G}}_{b}$, and since by Lemma $4, \Phi(N, W) \subseteq$ $C(N, W)$ for every $(N . W) \in \widetilde{\mathcal{G}}_{b}$, we have a contradiction to Axiom 6 . We conclude that $x \in \operatorname{Asp}(N, v)$.

Thus far we have obtained that the solution concept which satisfies our axioms must pick some subset of $\operatorname{Asp}(N . v)$. To get a characterization of $\mathrm{AC}(\cdot)$ we need to supplement by some conditions which guarantees that every element of $\operatorname{AC}(N, v)$ is indeed in $\Phi(N, v)$. An obvious approach consists in adding a converse of Axiom 6 to our list:

Axiom 7. Let $\Phi$ be a solution concept on $\mathcal{G}^{\prime} \cup \widetilde{\mathcal{G}}_{b}^{\prime}$ with $\mathcal{G}_{b} \subset \mathcal{G}^{\prime}$. If $(N, v) \in \mathcal{G}$ and $x$ is a payoff vector in $(N, v)$ such that $\left(x, x_{*}\right) \in \Phi\left(N, W_{d, v}\right)$, then $x \in \Phi(N, v)$.

We can now derive the final version of our result.

THEOREM 2. Let $\Phi$ be a solution concept on $\mathcal{G} \cap \widetilde{\mathcal{G}}_{b}$ which satisfies Axioms 1-7. Then $\Phi(N ., v)=A C(N, v)$ on each $(N, v) \in \mathcal{G}$.

Proof. From Theorem 1, we have that $\Phi(N, v) \subseteq \mathrm{AC}(N, v)$ for every $(N, v) \in \mathcal{G}$. Let $(N, v) \in \mathcal{G}$ and $x \in \mathrm{AC}(N, v)$ be arbitrary; by Lemma $6,\left(x, x_{*}\right) \in \mathrm{C}\left(N, W_{d}\right)$. Using Lemma 5 , we have that $\left(x, x_{*}\right) \in \Phi\left(N, W_{d}\right)$, and by Axiom 7 , we have that $x \in \Phi(N, v)$.

6. Concluding remarks. In this paper we have proposed a system of axioms characterizing the aspiration core, which selects those elements of the aspiration set which has smallest distance to the set of aggregate feasible payoff vectors. In this axiomatization, we have used axioms each of which is well-known and much used. However, we have used them in a context which is not quite commonplace; instead of reducing the number of players and applying a consistency axiom to the smaller game, we have transformed the game to another one, which may have as many players as the original game and which 
may not even be a TU game. However, further reduction in this class of games will work just as it does within the class of TU games, and the extension is anyway not very drastic, since we add only games which look as TU games for all coalitions except the grand coalition.

On the other hand, though it may be argued that NTU games are more basic than TU games so that involving some games which retain a certain degree of non-transferability of utility is permitted, it still would seem more appealing if the the axiomatization of a solution concept for TU games does not involve extensions of the solution to games that do not belong to this class. A consideration of such axioms is however a task for future research.

\section{References}

[1] E. Bennett, The aspiration approach to predicting coalition formation and payoff distribution in sidepayment games, International Journal of Game Theory 12 (1983), 1-28.

[2] O. N. Bondareva, Some applications of linear programming methods to the theory of cooperative games, Problemy Kibernetiki 10 (1963), 119-139 (in Russian).

[3] J. C. Gomez, An extension of the core solution concept, Discussion Paper 04-01, Institute of Economics, University of Copenhagen, 2004.

[4] J. C. Gomez, Axiomatizing a nonempty core extension, Mimeo, Institute of Economics, University of Copenhagen, 2004.

[5] T. Hokari and O. Kıbrıs, Consistency, converse consistency, and aspirations in TU games, Mathematical Social Sciences 45 (2003), 313-331.

[6] Y. Hwang and P. Südhölter, Axiomatizations of the core on the universal domain and other natural domains, International Journal of Game Theory 29 (2000), 597-623.

[7] B. Moldovanu and E. Winter, Consistent demands for coalition formation, in: Essays in Game Theory in Honor of Michael Maschler, Springer, New York, 1994, 129-140.

[8] B. Peleg, An axiomatization of the core of cooperative games without side payments, Journal of Mathematical Economics 14 (1985), 203-214.

[9] B. Peleg and P. Südhölter, Introduction to the Theory of Cooperative Games, Kluwer Academic Publishers, Boston, 2003.

[10] H. Scarf, The core of an n-person game, Econometrica 35 (1967), 50-97.

[11] L. S. Shapley, On balanced sets and cores, Naval Research Logistics Quarterly 14 (1967), 453-460. 